which $K=l$, the total variation is $J_{\bar{c}}-J_{\bar{c}_{0}}$. It is the object of Dr. Crathorne's paper to express this total variation in a form somewhat analogous to the Weierstrassian $E$-function representation for the simple calculus of variations problem.

H. E. Slaught, Secretary of the Section.

\title{
CONCERNING TWO RECENT THEOREMS ON IMPLICIT FUNCTIONS.
}

BY DR. LLOYD L. DINES.

(Read before the American Mathematical Society, October 26, 1912.)

THE theorems here considered are two recent generalizations of the Weierstrassian implicit function theorem,* by Professor G. A. Bliss† and Mr. G. R. Clements. $\ddagger$ They will be referred to respectively as Theorem B, and Theorem C.

The two theorems are similar in that they both give information concerning the number and character of the solutions of a system of equations

$$
f_{i}\left(x_{1}, \cdots, x_{n} ; y_{1}, \cdots, y_{p}\right)=0 \quad(i=1,2, \cdots, p)
$$

in the neighborhood of a point at which the functional determinant vanishes. They are different in that the assumptions concerning the functions $f_{i}$ are different. As is so often the case with similarly related theorems, the ranges of applicability overlap, but neither is wholly contained in the other.§ The purpose of this note is to characterize explicitly the four classes of cases: (I) in which neither theorem is applicable; (II) in which both theorems are applicable; (III) and (IV) in which one theorem is applicable while the other is not.

* Weierstrass, Abhandlungen aus der Funktionenlehre, p. 107.

$\dagger$ Bliss, "A generalization of Weierstrass' preparation theorem for a power series in several variables," Transactions, vol. 13, pp. 133-45 (April, 1912).

$\ddagger$ Clements, "Implicit functions defined by equations with vanishing Jacobian" (Theorem IV), Bulletin, vol. 18, p. 453 (June, 1912).

$\S$ In presenting this note to the Society, I made the statement that Mr. Clements's theorem was a corollary of Professor Bliss's. That this statement was incorrect was pointed out to me by Mr. Clements, who exhibited a numerical example in which the hypothesis of his theorem was satisfied while that of Professor Bliss was not. The example comes under Case IV as treated in this paper. 
In both theorems the functions $f_{i}$ are supposed to be analytic in a neighborhood of the point in question, and that point is without loss of generality assumed to be the origin $(x)=0$, $(y)=0$. Each of the functions is therefore represented in a certain neighborhood of the origin by a convergent power series in $x_{1}, \cdots, x_{n} ; y_{1}, \cdots, y_{p}$, with no constant term. If in any one of the series $f_{i}$ the variables $x_{1}, \cdots, x_{n}$ are put equal to zero, the result is a power series in $y_{1}, \cdots, y_{p}$ with no constant term. For the discussion which follows we will adopt the notation

$$
\begin{aligned}
f_{i}\left(0, \cdots, 0 ; y_{1}, \cdots, y_{p}\right) \equiv f_{i}{ }^{\left(k_{i}\right)}\left(y_{1}, \cdots, y_{p}\right) & \\
& +f_{i}{ }^{\left(k_{i}+1\right)}\left(y_{1}, \cdots, y_{p}\right)+\cdots,
\end{aligned}
$$

where $f_{i}^{(g)}\left(y_{1}, \cdots, y_{p}\right)$ represents a homogeneous polynomial of degree $g$ in $y_{1}, y_{2}, \cdots, y_{p}$. The leading polynomial $f_{i}{ }^{\left(k_{i}\right)}\left(y_{1}, \cdots, y_{p}\right)$ has been called by Bliss (following MacMillan), the characteristic polynomial of the series $f_{i}$. The resultant of the system of $p$ homogeneous polynomials $f_{i}{ }^{\left(k_{i}\right)}$ will be denoted by $R\left[f_{1}{ }^{\left(k_{1}\right)} f_{2}{ }^{(k 2)} \cdots f_{p}{ }^{\left(k_{p}\right)}\right]$.

With these conventions, the hypotheses of the two theorems in question can be stated as follows:

Hypothesis of Theorem $B$ :

(B) $\quad R\left[f_{1}{ }^{\left(k_{1}\right)} f_{2}{ }^{\left(k_{2}\right)} \cdots f_{p}{ }^{\left(k_{p}\right)}\right] \neq 0$, and $k=k_{1}, k_{2}, \cdots, k_{p}$.

Hypothesis of Theorem $C$ :

(C) $J_{k-1} \equiv \frac{D\left(J_{k-2}, f_{2}, \cdots, f_{p}\right)}{D\left(y_{1}, y_{2}, \cdots, y_{p}\right)}=0 \quad$ when $(x)=0,(y)=0$,

$$
J_{k} \equiv \frac{D\left(J_{k-1}, f_{2}, \cdots, f_{p}\right)}{D\left(y_{1}, y_{2}, \cdots, y_{p}\right)} \neq 0 \quad \text { when }(x)=0,(y)=0 .
$$

The conclusions of the two theorems may for our purpose be considered equivalent. Roughly speaking, both state that in the neighborhood of $(x)=0$, equations (1) define $y_{1}$, $y_{2}, \cdots, y_{p}$ as $k$-valued algebroid functions of $x_{1}, x_{2}, \cdots, x_{n}$, vanishing when $(x)=0 . *$

* Theorem $B$ states somewhat more than this, Theorem $C$ somewhat less. However this conclusion can be obtained from conditions $(C)$. 
In order to make clear the relations between the hypotheses $(B)$ and $(C)$ and thereby the relations between the corresponding theorems, we fix attention on two simple conditions $(b)$ and $(c)$, which seem to contain the primary restrictions of conditions $(B)$ and $(C)$ respectively; they are:

$$
\begin{aligned}
& R\left[f_{1}{ }^{\left(k_{1}\right)} f_{2}{ }^{\left(k_{2}\right)} \cdots f_{p}{ }^{\left(k_{p}\right)}\right] \neq 0, \\
& k_{2}=k_{3}=\cdots=k_{p}=1 .
\end{aligned}
$$

In terms of these two conditions, the ranges of applicability of Theorems $B$ and $C$ are sharply defined by the following statements:

I. In case neither $(b)$ nor $(c)$ is satisfied, neither Theorem B nor Theorem $C$ is applicable.

II. In case both (b) and (c) are satisfied, both Theorem $B$ and Theorem $C^{*}$ are applicable.

III. In case (b) is satisfied while (c) is not, Theorem B is applicable while Theorem $C$ is not.

IV. In case (b) is not satisfied while (c) is satisfied, Theorem B is not applicable; but Theorem $C$ may be, and is applicable if and only if there is an integer $h\left(>k_{1}\right)$ such that $J_{h}(0, \cdots, 0 ; 0, \cdots, 0) \neq 0$.

The truths of these statements follow easily from two lemmas which we now prove.

Lemma I: Conditions $(C)$ can be satisfied only when $k_{2}=k_{3}$ $=\cdots=k_{p}=1$.

By definition

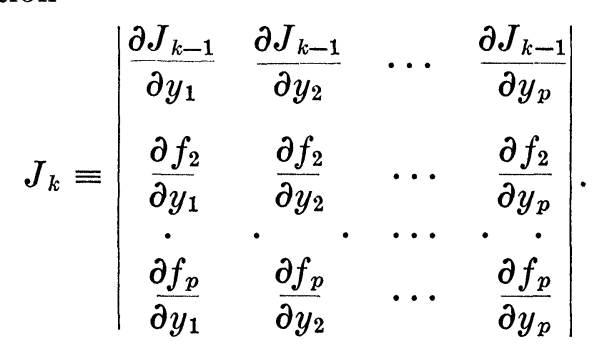

Suppose now that one of the indices $k_{2}, k_{3}, \cdots, k_{p}$ is greater than 1. We may assume without loss of generality that it is $k_{2}$. Then the series $f_{2}\left(0, \cdots, 0 ; y_{1}, y_{2}, \cdots, y_{p}\right)$ begins with

* The fact included in II that when $(b)$ and $(c)$ are satisfied the hypotheses $(C)$ are satisfied furnishes a generalization of Theorem VI of Mr. Clements's paper. 
terms of higher degree than the first. Therefore all the elements in the second row of the determinant $J_{k}$ must when $(x)=0$ begin with terms of at least the first degree in $y_{1}$, $y_{2}, \cdots, y_{p}$, and hence when $(x)=0$ and $(y)=0$, the determinant $J_{k}$ must vanish and $(C)$ cannot be satisfied.

Lemma II: If $k_{2}=k_{3}=\cdots=k_{p}=1$, then $J_{g}(0, \cdots, 0$; $0, \cdots, 0)=0$ when $g<k_{1}$, and the condition $J_{k_{1}}(0, \cdots, 0$; $0, \cdots, 0) \neq 0$ is equivalent to the condition $R\left[f_{1}{ }^{\left(k_{1}\right)} f_{2}{ }^{(1)} \cdots f_{p}{ }^{(1)}\right] \neq 0$.

Since $J_{g}$ is by definition a determinant in which the elements are power series, it is itself a power series, and we may use the notation

$$
J_{g}\left(0, \cdots, 0 ; y_{1}, \cdots, y_{p}\right) \equiv J_{g}^{\left(j_{g}\right)}+J_{g}^{\left(j_{g}+1\right)}+\cdots,
$$

where $J_{g}(h)$ is a homogeneous polynomiäl of degree $h$. For the purpose of determining the value of $J_{g}(0, \cdots, 0 ; 0, \cdots, 0)$, it is sufficient to compute the leading polynomial $J_{g}^{\left(j_{g}\right)}\left(y_{1}, \cdots, y_{p}\right)$. The hypothesis $k_{2}=k_{3} \cdots=k_{p}=1$ makes this computation simple.

From the definition of $J_{1}$ it follows that the first polynomial which can be different from zero in $J\left(0, \cdots, 0 ; y_{1}, \cdots, y_{p}\right)$ is

$$
\left|\begin{array}{cccc}
\frac{\partial f_{1}^{\left(k_{1}\right)}}{\partial y_{1}} & \frac{\partial f_{1}^{\left(k_{1}\right)}}{\partial y_{2}} & \ldots & \frac{\partial f_{1}\left(k_{1}\right)}{\partial y_{p}} \\
a_{21} & a_{22} & \ldots & a_{2 p} \\
a_{31} & a_{32} & \ldots & a_{3 p} \\
\cdot & \cdot & \cdots & \cdot \\
a_{p 1} & a_{p 2} & \cdots & a_{p p}
\end{array}\right|
$$

where $a_{i j}=\partial f_{i}{ }^{(1)} / \partial y_{j}$, that is a constant, since $f_{i}{ }^{(1)}$ is a linear form. Since $f_{1}{ }^{\left(k_{1}\right)}$ is of degree $k_{1}$, this determinant represents a polynomial of degree $k_{1}-1$. It can be expanded in the form

$$
J_{1}{ }^{\left(k_{1}-1\right)} \equiv \frac{\partial f_{1}{ }^{\left(k_{1}\right)}}{\partial y_{1}} A_{1}+\frac{\partial f_{1}{ }^{\left(k_{1}\right)}}{\partial y_{2}} A_{2}+\cdots+\frac{\partial f_{1}{ }^{\left(k_{1}\right)}}{\partial y_{p}} A_{p},
$$

where $(-1)^{j-1} A_{j}$ is equal to the determinant obtained from the matrix

$$
\left\|\begin{array}{cccc}
a_{21} & a_{22} & \cdots & a_{2 p} \\
a_{31} & a_{32} & \cdots & a_{3 p} \\
\cdot & \cdot & \cdots & \cdot \\
a_{p 1} & a_{p 2} & \cdots & a_{p p}
\end{array}\right\|
$$

by striking out the $j$ th column. 
The first polynomial of $J_{2}\left(0, \cdots, 0 ; y_{1}, \cdots, y_{p}\right)$ which can be different from zero may be computed as follows:

$$
\begin{aligned}
J_{2}{ }^{\left(k_{1}-2\right)} & \equiv\left|\begin{array}{cccc}
\frac{\partial J_{1}{ }^{\left(k_{1}-1\right)}}{\partial y_{1}} & \frac{\partial J_{1}{ }^{\left(k_{1}-1\right)}}{\partial y_{2}} & \ldots & \frac{\partial J_{1}{ }^{\left(k_{1}-1\right)}}{\partial y_{p}} \\
a_{21} & a_{22} & \ldots & a_{2 p} \\
a_{31} & a_{32} & \ldots & a_{3 p} \\
\cdot & \cdot & \cdots & \cdot \\
a_{p 1} & a_{x_{2}} & \cdots & a_{p p}
\end{array}\right| \\
& \equiv \frac{\partial J_{1}{ }^{\left(k_{1}-1\right)}}{\partial y_{1}} A_{1}+\frac{\partial J_{1}{ }^{\left(k_{1}-1\right)}}{\partial y_{2}} A_{2}+\cdots+\frac{\partial J_{1}{ }^{\left(k_{1}-1\right)}}{\partial y_{p}} A_{p} \\
& \equiv \sum_{i=1}^{p} A_{i} \frac{\partial}{\partial y_{i}} \sum_{j=1}^{p} \frac{\partial f_{1}{ }^{\left(k_{1}\right)}}{\partial y_{j}} A_{j} \equiv \sum_{i=1}^{p} \sum_{j=1}^{p} \frac{\partial^{2} f_{1}{ }^{\left(k_{1}\right)}}{\partial y_{i} \partial y_{j}} A_{i} A_{j} \\
& \equiv\left[\frac{\partial f_{1}{ }^{\left(k_{1}\right)}}{\partial y_{1}} A_{1}+\frac{\partial f_{1}{ }^{\left(k_{1}\right)}}{\partial y_{2}} A_{2}+\cdots+\frac{\left.\partial f_{1} k_{1}\right)}{\partial y p} A_{p}\right]^{(2)} .
\end{aligned}
$$

A simple mathematical induction gives for the first polynomial of $J_{g}\left(0, \cdots, 0 ; y_{1}, \cdots, y_{p}\right),\left(g \leqq k_{1}\right)$ which can be different from zero, the formula

(3) $J_{g}{ }^{\left(k_{1}-g\right)} \equiv\left[\frac{\partial f_{1}^{\left(k_{1}\right)}}{\partial y_{1}} A_{1}+\frac{\partial f_{1}{ }^{\left(k_{1}\right)}}{\partial y_{2}} A_{2}+\cdots+\frac{\partial f_{1}^{\left(k_{1}\right)}}{\partial y_{p}} A_{p}\right]^{(g)}$.

If $g<k_{1}$, this polynomial is of first or higher degree and therefore $J_{g}(0, \cdots, 0 ; 0, \cdots, 0)=0$; and the first part of the lemma is proved.

If $g=k_{1}$, formula (3) gives the constant term of $J_{k}^{1}$. Since $f_{1}{ }^{\left(k_{1}\right)}$ is homogeneous of degree $k_{1}$, we have by Euler's theorem

$$
\begin{aligned}
& {\left[\frac{\partial f_{1}^{\left(k_{1}\right)}}{\partial y_{1}} A_{1}+\frac{\partial f_{1}^{\left(k_{1}\right)}}{\partial y_{2}} A_{2}+\cdots \frac{\partial f_{1}^{\left(k_{1}\right)}}{\partial y_{p}} A_{p}\right]^{\left(k_{1}\right)}} \\
& =k_{1} ! f_{1}{ }^{\left(k_{1}\right)}\left(A_{1}, A_{2}, \cdots, A_{p}\right) \text {, }
\end{aligned}
$$

and therefore

$$
\text { (4) } J_{k_{1}}(0, \cdots, 0 ; 0, \cdots, 0)=k_{1} ! f_{1}{ }^{\left(k_{1}\right)}\left(A_{1}, A_{2}, \cdots, A_{p}\right) \text {. }
$$

In order to complete the proof of the lemma, it is sufficient, on account of (4), to show that $f_{1}{ }^{\left(k_{1}\right)}\left(A_{1}, A_{2}, \cdots, A_{p}\right)=0$ when and only when $R\left[f_{1}{ }^{\left(k_{1}\right)} f_{2}{ }^{(1)} \cdots f_{p}(1)\right]=0$. Consider the 
system of homogeneous equations

$$
\begin{aligned}
f_{1}{ }^{\left(k_{1}\right)}\left(y_{1}, y_{2}, \cdots, y_{p}\right) & =0, \quad(i=2,3, \cdots, p) . \\
f_{i}{ }^{(1)}\left(y_{1}, y_{2}, \cdots, y_{p}\right) & =0
\end{aligned} \quad(i=
$$

The last $p-1$ of these equations are linear and the matrix of their coefficients is (2). Two cases are to be considered according as the rank of this matrix is less than or equal to $p-1$.

If the matrix (2) is of rank less than $p-1$, both $f_{1}{ }^{\left(k_{1}\right)}\left(A_{1}, A_{2}, \cdots, A_{p}\right)$ and $R\left[f_{1}{ }^{\left(k_{1}\right)} f_{2}{ }^{(1)} \cdots f_{p}^{(1)}\right]$ are equal to zero; the former because $A_{1}=A_{2}=\cdots=A_{p}=0$, the latter because the polynomials $f_{2}{ }^{(1)}, f_{3}{ }^{(1)}, \cdots, f_{p}(1)$ are not independent.

If the matrix (2) is of rank $p-1$, then the solution of the last $p-1$ equations of (5) is

$$
y_{j}=\rho A_{j} \quad(j=1,2, \cdots, p),
$$

$\rho$ being an arbitrary factor of proportionality. Therefore a necessary and sufficient condition that the system of $p$ equations (5) have a solution in which not all of the variables are zero, is $f_{1}{ }^{\left(k_{1}\right)}\left(A_{1}, A_{2}, \cdots, A_{p}\right)=0$. But it is well known that a necessary and sufficient condition for the existence of such a solution is $R\left[f_{1}{ }^{\left(k_{1}\right)} f_{2}{ }^{(1)} \cdots f_{p}{ }^{(1)}\right]=0$. Hence the two conditions are equivalent, and from (4) follows the desired conclusion of the lemma.

The statements I-IV will now be proved.

Proof of $I$ : In case neither $(b)$ nor $(c)$ is satisfied: Theorem $B$ is not applicable, since its hypothesis includes $(b)$; and Theorem $C$ cannot be applicable, by Lemma I.

Proof of II: In case both $(b)$ and $(c)$ are satisfied, both Theorems $B$ and $C$ are applicable. The hypothesis $(B)$ is evidently satisfied, $k$ being equal to $k_{1}$. And $(C)$ is also satisfied, $k$ being equal to $k_{1}$, as a direct consequence of Lemma II.

Proof of III: Since $(b)$ is satisfied, $(B)$ is satisfied, $k$ being equal to $k_{1} k_{2} \cdots k_{p}$. Since $(c)$ is not satisfied, $(C)$ cannot be satisfied, by Lemma I.

Proof of $I V$ : Since $(b)$ is not satisfied $(B)$ cannot be. Evidently $(C)$ is satisfied if and only if there exists an integer $h$ such that $J_{h}(0, \cdots, 0 ; 0, \cdots, 0) \neq 0, k$ then being the smallest such integer. That $k$ is necessarily greater than $k_{1}$ follows from Lemma II, and the fact that $(b)$ is not satisfied. 